\title{
Identification and Counting of Fertile Pollen Grains using Morphological operators, FSF and CGF
}

\author{
R.S.Sabeenian \\ Professor-ECE and Centre Head \\ Sona SIPRO \\ Advanced Research Centre, Sona \\ College of Technology, Salem - \\ 636005, Tamil Nadu, INDIA
}

\author{
M.E.Paramasivam \\ Assistant Professor -ECE\& \\ Research Staff - SONA SIPRO \\ Advanced Research Centre, Sona \\ College of Technology, Salem - \\ 636005, Tamil Nadu, INDIA
}

\author{
P.M. Dinesh \\ Assistant Professor -ECE\& \\ Research Staff - SONA SIPRO \\ Advanced Research Centre, Sona \\ College of Technology, Salem - \\ 636005, Tamil Nadu, INDIA
}

\begin{abstract}
The pollen grain in a plant is apart used to transport the male gamete (i.e. male DNA) to the female part of a flower. There are two types of pollen grains-fertile and sterile. Only the fertile pollen grains provide good yield. The scope of the work is to identify the fertile pollen grains using Morphological operators along with FSF and CGF. This identification can be done using two approaches-based on intensity and size variation. The Proposed work mainly focuses on the identification of fertile pollen grains based on size variation. When we look into the pollen images, the fertile ones appear larger than the sterile. Thus, by finding the size of each pollen grain we can differentiate the fertile and sterile pollen grains. In order to find the size of the pollen grains we go in for approximate radius calculation. We have developed a Graphical User Interface using MATLAB for identification and counting of the fertile pollen grains which can be used in research centers
\end{abstract}

\section{Keywords:}

Fertile, Sterile, Microgametes, Intensity Variation, Pollen Grains, FSF (First order Statistical Features), CGF (Common Geometrical Features), Morphological operators

\section{INTRODUCTION}

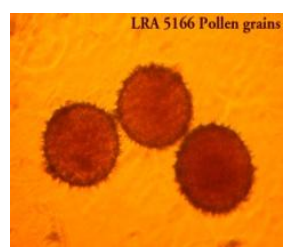

Fig 1:Pollen Grains

Pollen (see Figure 1) [1] is produced in the male organs of the flowers - anthers. Pollination occurs when pollen is transferred from the anthers to the female organs by wind (anemophily) or by animals (zoophyly).

For feminization the central cytoplasmic part is the main source. An inner part, the inner and outer layer, the extine are the other parts acts as wall for the grain. The least inner part of cellulose is the intine. The other layers act as protective wall from disintegration. The composition of the exine is uncertain; its constituents have been termed sporopollenins.

Ecological information transfer and regulation of plasticity in cross pollination is done by the transferred quality of the pollen. Over flowing pollen shows the finest eco-friendly condition ( for example consider a plant is located at the center of natural value, in idyllic growing condition, has bulk or large in number of male plants adjacent and positive weather condition). But small quantity of pollen specifies extreme conditions (for example, consider a plant is located at boundary of natural value, with a shortage of male plants, and antagonistic climate conditions).

There are two types of pollen grains:

- Fertile

- Sterile

Merely the fruitful pollen grains deliver respectable yield. The possibility of the work is to identify the fruitful pollen grains using statistical and common geometric features. This identification can be done using two approaches-based on:

- Intensity variation (Parameters)

- Size

This work mainly focuses on the identification of fertile pollen grains based on size variation. When we look into the pollen images, the fertile ones appear larger than the sterile. Thus, by finding the size of each pollen grains we can differentiate the fertile and sterile pollen grains. In order to find the size of the pollen grains we go in for approximate radius calculation.

\section{PREVIOUS METHODS}

2.1 Detection and Classification of Grains of Pollen Based on Shape and Texture

The shapes of pollens are nearly circle in shape. The pollens can be identified by the circle detection process. In image processing the Hough transformation method is used to find the circles[2][3]. It converts the edge pixels in to conic surface, so circle is formed in edge images. The Hough transform is done with three major factors like Radii, Threshold values and Binary Edge input.

\subsection{Photoacoustic Microscope Method}

A method with photo thermal effect can be called as photo acoustic micro scope method [PAM]. The light photons are spotted over a sample is converted in to thermal part image. Most of light photons form modulation light source. The photo thermal effect can be used to find the presences and quantity of the pollen in give sample. The photo thermal image can prove in counting cryptometria japonica pollen [4]. The photo acoustic signals are formed by the surface of the specimen. These signals depend on the quality and presents of the pollen. 


\subsection{Automatic Recognition of Biological Particles in Microscopic Images}

At first the pollen area of the image is found and for that small region samples are generated. This is done with feature finder. Next differential brightness is calculated in many resolutions of the samples [5] [6]. Now the point wise mapping is done to higher feature space. The mapped information is averaged. The averaged result produces a vector of feature for each sample. Now classifier is used to classify the pollen samples

\subsection{Manual Counting of pollen using Microscopic Images}

Techniques for counting pollen vary in part based on grain size and number of grains produced per anther. A small subset of the total pollen, such as four fields of view at medium power (about 100x) under a compound microscope, can be counted and used to find the total number of pollen grains in the sample[7].

The field of view is the area viewed under medium power $(100 \times)$ with a compound microscope; it is calculated after measuring the diameter with a stage micrometer. Then the diameter of the dish can be measured with callipers and its area calculated. Dish area and field of view area can be calculated as follows

Pollen Grains per dish =

(Total grains per field view Count) X (Petri dish area)

Total area of view counted

Pollen Grains per Flower =

(Grains per dish) X (number of stamems)

The pollen count is a measure of the number of pollen grains of a certain type per cubic meter of air sampled, averaged over 24 hours. Counting each slide can take an hour or even longer depending on the amount of pollen on it. Nobody has yet developed a satisfactory automatic method for counting pollen.

\section{FEATURE EXTRACTION}

\subsection{Statistical Features.}

1. Energy: The energy value of the image is defined as the sum of the square of image pixel value [8-12].

$$
f_{E}=\sum_{i, j} \hat{f}(i, j)^{2}
$$

2. Mean:The mean value of the image is defined as the average value of the image gray level.

$$
\text { Mean }=\sum_{i} \sum_{j} f(i, j) / N
$$

3.The standard deviation (SD) gives us a convenient measure of the "spread" of a set of random data items. It can be computed using the formula$$
\mathrm{SD}=\sum_{i} \sum_{j}\left((f(i, j)-\mu)^{2} / N\right)^{1 / 2}(3)
$$ \\ 3.2 Common Geometrical Features $(C G F)$ :}

1. Area $(A)$ : The area of an object $S$ is defined as the number of internal points of $S$.
2. The perimeter $(P)$ of an object $S$ can be defined as thearc length of its contour[13 \& 14].

3. Ratio of area and perimeter (RAP): This parameter is the ratio between the area and the perimeter $(\mathrm{RAP}=A / P)$.

4. Diameter $(D): D$ is the largest distance between any two points on the boundary of the object.

5. Compactness $(C)$ : The compactness is calculated as

$C=P 2 /(4 \pi A)$

6. Roundness $(R)$ : It is the inverse of the compactness $R=1 / C$

7. Thickness $(T)$ : The thickness $T$ is the number of times that the binary object can be eroded by a $3 \times 3$ squared filter mask until it vanishes [13 \& 14].

8. Eccentricity $(E c)$ : The eccentricity estimates the ratio of the major axis and theminor axis of the best fit ellipse over the object. The axes are determined by the second-order central moments of the binary shape $S$

\section{PROPOSED ALGORITHM}

Till date, the counting using microscope is done manually which is time consuming. All the measurements and parameter calculations are done only manually Calculation of the grain radius depends on the accuracy of fixing the centre of the pollen and fine tunings of the microscope scales. This is a time consuming and hectic process. It involves a lot of human labor with technical skills and experience.

The best technique for pollen counting would be to take a picture of all the pollen grains in the entire Petri dish and extract both statistical features and common geometrical features(CGF) and quantify them using a computer program. A GUI has been developed using MATLAB for identifying and counting of the fertile pollen grains.

The advantage of theGUI is that the process of finding the size and counting the pollen grains is fully automated and no human intervention is required for any intermediate calculations.

The time required for the algorithm is also reduced and the technique used is very simple. The radius estimation involves simple analytical formulae and the algorithm readily displays the radius within a few seconds.

Only the fertile pollen grains are identified which are larger in size and the radius is estimated. Similarly, the pollen count is given only for the fertile pollen grains. Thus, the algorithm can be used to segregate the fertile and the sterile pollens.

\section{POLLEN IDENTIFICATION}

In this Process the input image is acquired and is a function of reflectance and luminance, generally given as $f(x, y)$. The input image acquired may be of any irregular size. So, in order to acquire a regular size the image is resized to the size of 256X256. The resized input image has different colored pixels values (RGB) and hence for further processing we convert them to Gray level values as shown Figure (see Figure 2).

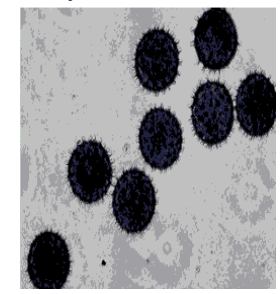

Figure 2:Gray scale Image of Original image 
The background details of the image are to be suppressed and hence all the background pixels are whitened by equating the pixel values greater than the threshold value to 255.Those pixel values less than the threshold are equated to zero. For enhancing the fine edge details of the pollen grains we do edge detection using Robert Cross filter.

Despite accessing the horizontal and vertical details it is possible to obtain the diagonal details also effectively. Having this advantage we use this filter in our algorithm. The sensitivity threshold for edge detection is determined. "Edge" ignores all edges that are not stronger than threshold value. For further enhancement of the edge detection the threshold is multiplied with the multiplication factor. The value of the multiplication factor can be chosen on the type of image.

Image dilation is done to fill the discontinuities on the boundaries of the pollens. In this step the image is dilated by using the structuring element object, or array of structuring element objects, returned by the "strel" function. Using "strel" command we create a morphological structuring element "line" a flat, linear structuring element, with length as 3 and degrees 90 and 0 , as measured in a counter clockwise direction from the horizontal axis.

After image dilation, still some holes are present inside the pollen grains. So, the inner gaps are filled using filling command. In the image, some of the pollen grains are found to be connected at the border of the image. These pollens are removed from the image using clear border function. The connectivity parameter is set to remove diagonal connections.

The pollen is almost circle in shape and hence we trace its boundary by using boundary trace function. The row and column dimensions are determined.

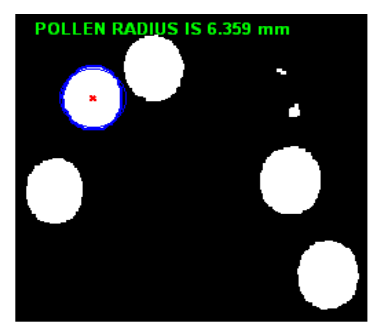

Figure 3: Pollen Radius

The direction of traversal is assumed to be 'east' and traversal is in 'counter clockwise' motion. The initial step is assumed to be ' 180 'degrees and ' 8 ' connectivity is chosen. Hence the outline of the pollenis drawn. So now we can find the Radius of the pollens as show in the Figure(see Figure 3).

\section{COUNTING THE NUMBER OF POLLEN}

Similar to the process of finding the radius of the pollen, the initial steps of edge detection, image dilation and clearing the connected objects in the border is done as shown in Figure(see figure 4). All connected components (objects) that have fewer than 200 pixels, are removed from the image. Eight connectivity is used here.

Now, we create a morphological structuring element "line" a flat, disk-shaped structuring element, with radius as three. Using this structure element we erode the image twice. Those pollens with pixels less than 200(i.e., sterile) are suppressed.

Thus we segment the fertile pollens separately. The outline of the each pollen is obtained by drawing the perimeter of the image.Perimeter command is used for extracting only the border pixels of each pollen. This outline alone is now whitened for display.

Each pollen is labeled with individual numbers. Using labeling command the connected objects in a binary image can be labeled. As we have the entire outline of each pollen we can label them using this command.

The pollens are labelled and now they should be displayed as against the background. The labels are in a series of numbers and thus they represent the pollen count as shown in Figure (see Figure 4). To perform labelling, the background is blackened and only the pollen outlines are whitened. Properties of the pollen on each extreme are obtained using region property command. Elements number finder function returns the number of elements in array or subscripted array expression.

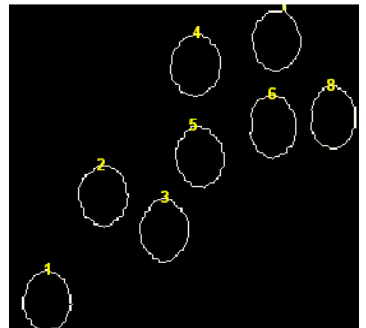

Figure 4: Pollen Count

Each pollen is recognized as an element in an array and hence the number of pollens could be obtained using the Elements number finder function. This value is traversed through a loop and the count for each pollen is thus displayed. This display is done using display command. The display can be varied by changing the font weight, font style, markers, pointers, coolers, backgrounds for enhance effects

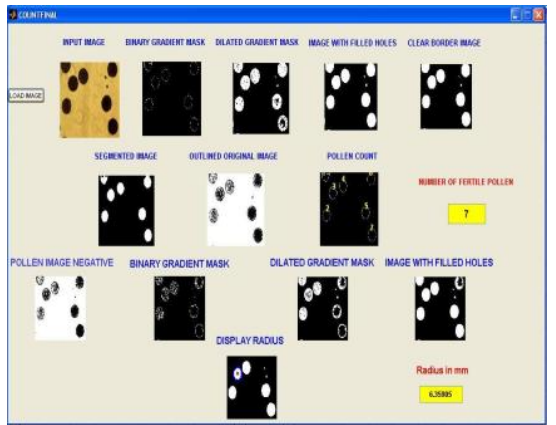

Figure 5: GUI Representation for Pollen Count and Radius of Pollen 
Thus we have developed an automated tool for the identification of fertile pollen grains and calculating their size (radius). The developed GUI (Graphical user Interface) is shown in the Figure (see Figure 5). This GUI Shows the Number of fertile pollens that present in image and size of the same is calculated

\section{APPLICATIONS}

The proposed algorithm is simple, cost effective and portable. So it can be easily implemented in pollen research institutes. It can also be used in biological research institutes and museums for database maintenance.

\section{CONCLUSION}

This automated GUI is cost efficient and does not involve any manual work unlike normal microscopic measurements. The GUI for counting the pollen grains is time saving and effective method and could be used for many research purposes. The proposed method proves to be more effective and compared to the traditional methods.

\section{FUTURE WORKS}

We have developed a simple technique for identifying and counting the fertile pollen grains. Further analysis and research can be done by using transforms like Gabor transform, curvelet transform. Effective segmentation could be achieved by using techniques like watershed transform. An automatic pollen counting system could be developed with the help of a robot traversing the pollen slides and suitable hardware being implemented.

Currently research work is being done to find pollen size automatically by using transforms like Wavelet transform. As a further enhancement, our algorithm could be improvised by using such transforms like Contourlet and Ridgelet.

\section{REFERENCES}

[1] WenqingJia; Huichao Liu; Xiaohua Du; XiaoboXu; Yang You; Determination of Jasminumnudiflorum pollen viability and its storage method, Proceedings of IEEE International Conference on Remote Sensing, Environment and Transportation Engineering (RSETE), 2011.

[2] Brau, E.; Barnard, K.; Palanivelu, R.; Dunatunga, D.; Tsukamoto, T.; Lee, P.; A generative statistical model for tracking multiple smooth trajectories, Proceedings of Computer Vision and Pattern Recognition (CVPR), 2011 IEEE Conference, June 2011.

[3] Mar'1aRodr'iguez-Dami'an, Eva Cernadas, Arno Formella, Manuel Fern'andez-Delgado, and Pilar De S'aOtero, Automatic Detection and Classification of Grains of Pollen Based on Shape and Texture, IEEE Transactions On Systems, Man, And Cybernetics-Part C: Applications And Reviews, Vol. 36, No. 4, July 2006

[4] Katsuhiko Miyamoto and Tsutomu Hoshimiya, Measurement of the Amount and Number of Pollen Particles of Cryptomeria japonica (Taxodiaceae) by Imaging with a Photoacoustic Microscope, ieee transactions on ultrasonics, ferroelectrics, and frequency control, vol. 53, no. 3, march 2006

[5] M. Ranzatoa,P.E. Taylor b J.M. House b R.C. Flagan b Y. LeCun a P. Perona b, Automatic Recognition of Biological Particles in Microscopic Images, Journal Pattern Recognition Letters, Volume 28 Issue 1, January,2007 Elsevier Science Inc

[6] Ronneberger, O.; Reusable modules for high-content 3D and 4D image analysis, Proceedings of Biomedical Imaging: From Nano to Macro, 2011 IEEE International Symposium, April 2011
[7] Seelamantula, C.S.; Pavillon, N.; Depeursinge, C.; Unser, M.; Zero-order-free image reconstruction in digital holographic microscopy, Proceedings of Biomedical Imaging: From Nano to Macro, 2009. ISBI '09. IEEE International Symposium, July 2009

[8] Sabeenian R.S. 'Texture Image Classification using Multi Resolution Combined Statistical and Spatial Frequency Method' Published in the International Journal of Technology And Engineering System (IJTES): Jan - March 2011- Vol.2, No.2, pp 167-171

[9] Sabeenian R.S. and Palanisamy V., 'Texture Based Weed Detection Using Multi Resolution Combined Statistical and spatial Frequency', Published in the International Journal of Information and Communication Engineering(ISSN 2070-3740), Vol. 52, pp. 549-553. April 2009.

[10] Sabeenian R.S. and Palanisamy V., 'Crop and Weed Discrimination in Agricultural Field using MRCSF' Published in the International Journal of Signal and Imaging Systems Engineering (IJSISE) Published by Inderscience Publishers,Vol.3,No.1,pp 61-69.

[11] Sabeenian R.S. and Palanisamy V., 'Texture Image Classification using Gabor Statistical Features (GSF) and Wavelet Statistical Features (WSF)' Published in the International Journal of Computer Science and Applications (ISSN No. 0974-0767) Vol.2 No.1, June 2010,pp 5-9.

[12] Sabeenian R.S. and Palanisamy V., 'Texture Based Medical Image Classification of Computed Tomography images using MRCSF', Published in International Journal of Medical Engineering and Informatics (IJMEI), Vol. 1, No. 4 pp. 459-472 July 2009 Published by Inderscience Publications.

[13] YonghuaXie; Ó hÉ igeartaigh , M.; 3D Pollen particle recognition based on spatial geometric constraints histogram descriptors, Image and Signal Processing (CISP), 2010 3rd International Congress, oct 2010

[14] Travieso, C.M.; Briceno, J.C.; Ticay-Rivas, J.R.; Alonso, J.B.; Pollen classification based on contour features, Proceedings of Intelligent Engineering Systems (INES), 2011 15th IEEE International Conference,2011

\section{AUTHORS PROFILE}

Dr.R.S.Sabeenian is currently working as an Professor in ECE Department in Sona College of Technology, Salem, Tamil Nadu, India. He received his Bachelors in Engineering from Madras University and his Masters in Engineering in Communication Systems from Madurai Kamaraj University. He received his Ph.D Degree from Anna University, Chennai in the area of image processing. He is currently heading the research group named Sona SIPRO (SONA Signal and Image PROcessing) centre located at the Advanced Research Centre in Sona College of Technology, Salem. His areas of interest include texture analysis, texture classification and pattern recognition. He has published around 33 papers in various international, national journals and conferences. He has also published around seven books. He is a reviewer for the journals of IET, UK and ACTA Press Singapore. He received the - Best Faculty Award among Tamil Nadu, Karnataka and Kerala states for the year 2009 given by the Nehru Group of Institutions, Coimbatore, "ISTE RajarambapuPatil National Award" for promising Engineering Teacher for Creative work done in Technical Education (Colleges) for the year 2010 and "IETE BimanBehariSen Memorial Award" for outstanding contributions in the emerging areas of Electronics and 
Telecommunication with emphasis on R\&D for the year 2011. $\mathrm{He}$ has also received a Project grant from the All India Council for Technical Education and from the Tamil Nadu State Council for Science and Technology for his research.

M.E.Paramasivam, is currently working as the Research Staff in Sona Signal and Image PROcessing Research Centre located at the Advanced Research Centre, Sona College of Technology, Salem. He is also the Assistant Professor in the ECE department of Sona College of Technology, Salem.

$\mathrm{He}$ completed his Bachelors in Electronics and Communication Engineering in the year 2005. Later he was selected on an all India basis for pursing the Advanced Post Graduate Diploma in VLSI Design, from VEDANT Center, of Semiconductor Complex Ltd., A Govt. of India Enterprise located at Chandigarh in the year 2006. He also holds a Diploma in Management, Post Graduate Diploma in Management, Post Graduate Diploma in Human Resource Management and Masters in Management. He is a certified Altera Tools trainer, certified by Altera Semiconductors. USA. He also holds a Masters Degree in Engineering with specialization in VLSI Design in the year 2011. He is currently pursuing his Doctoral Research in the area of Digital Image Processing. $\mathrm{He}$ has published and presented 15 research papers, both in technical and non-technical areas, at various conferences conducted around the nation. To his credit he has also published two papers in International Journal. He has written a book on "Electric Circuits and Electron Devices" in 2009.. He has also received a Project grant from the All India Council for Technical Education under the Research Promotion Scheme (RPS).

P.M.Dinesh is currently working as Assistant Professor of ECE Department, at Sona College of Technology, Salem. He is also a Team member of Sona SIPRO, Sona Signal and Image PROcessing Research Centre. He completed his Bachelors in Engineering in Electronics and Communication Engineering in the year 2005 and Masters in Engineering in VLSI Design in the year 2011. He is currently pursuing his Doctoral Research in the area of Image Processing. His areas of interest include pattern recognition and texture analysis using MATLAB. He has published ten papers in leading conferences and journals in the country. 